GLASNIK MATEMATIČKI

Vol. 40(60)(2005), $249-259$

\title{
UNSTABLE NEUTRAL DIFFERENTIAL EQUATIONS INVOLVING THE MAXIMUM FUNCTION
}

\author{
Guang Zhang and MaŁgorzata Migda \\ Qingdao Institute of Architecture and Engineering, P.R. China and \\ Poznań University of Technology, Poland
}

\begin{abstract}
A nonlinear differential equation involving the maximum function is studied. The existence and asymptotic behavior of nonoscillatory solutions are considered. The difference between the positive and negative solutions is illustrated by some examples. Oscillation of solutions is also studied.
\end{abstract}

\section{IntRoduction}

Nonlinear functional differential equations involving the maximum function are important since they appear naturally in automatic control theory (see e.g. Popov [1]). In this paper, we will be concerned with the following functional differential equation

$$
(x(t)-p x(t-\tau))^{\prime}=q(t) \max _{s \in[t-\sigma, t]} x(s), t \geq t_{0},
$$

where $\tau>0, \sigma \geq 0, p \in R$ and $q$ is a nonnegative and continuous function on $\left[t_{0}, \infty\right)$ and is not identically zero on any half line $[t, \infty)$. Oscillatory and asymptotic properties of this equation have been studied by a number of authors, see e.g. Bainov et al. [2], Zhang and Zhang [3], and Zhang and Cheng [4]. However, none of the previous work applies to equation (1) with $q(t) \geq 0$. Consequently, the results in this work are new.

By a solution of (1) we mean a function $x(t)$ which is defined for $t \geq$ $t_{0}-\max \{\tau, \sigma\}$ and which satisfies (1) for $t \geq t_{0}$. By the method of steps, we know that, for a given initial function $\varphi \in C\left(\left[t_{0}-\max \{\tau, \sigma\}, t_{0}\right], R\right)$, there

2000 Mathematics Subject Classification. 34K15.

Key words and phrases. Differential equation, maximum function, nonoscillation, oscillation, asymptotic property.

Supported by Natural Science Foundation of Qingdao Institute of Architecture and Engineering. 
exists a unique solution defined for $t \geq t_{0}-\max \{\tau, \sigma\}$ and which satisfies the initial condition for $t_{0}-\max \{\tau, \sigma\} \leq t \leq t_{0}$. Since our purpose is to discuss oscillatory and nonoscillatory solutions of (1), every solution $x(t)$ mentioned here will be understood to be nontrivial and continued to the right, i.e., $x(t)$ is defined on $\left[t_{x}, \infty\right)$ for some $t_{x} \geq t_{0}$ and $\sup \left\{|x(t)|: t \geq t_{1}\right\}>0$ for every $t_{1} \geq t_{x}$. Furthermore, we will say that such a solution is oscillatory if its set of zeros is unbounded from above, and say that it is nonoscillatory otherwise.

The plan of this paper is the following. In the next section, we will discuss the existence of nonoscillatory solutions of equation (1) for $p \geq 0$. In Section 3, some asymptotic properties are obtained. The difference between the eventually positive and negative solutions is illustrated by some examples. Finally in Section 4 we present some oscillation results.

For the sake of convenience, all inequalities are assumed to hold for all sufficiently large $t$.

\section{Existence of Nonoscillatory Solutions}

In this section, we give the existence and growth conditions of nonoscillatory solutions of equation (1). We begin with the following theorem.

THEOREM 2.1. Let $p \geq 0$. Then equation (1) has an eventually positive solution.

Proof. Choose a positive continuous function $H(t)$ such that

$$
\int_{t_{0}}^{\infty} q(t) H(t) d t=\infty
$$

and

$$
\lim _{t \rightarrow \infty}\left\{\frac{q(t)}{\exp \left(\int_{t_{0}}^{t} q(s) H(s) d s\right)}\right\}=0
$$

Define a function $v$ by

$$
v(t)=\exp \left[\int_{t_{0}}^{t} \exp \left(\int_{t_{0}}^{s} q(u) H(u) d u\right) d s\right] .
$$

Let $B C$ be the Banach space of all bounded and continuous functions $y$ : $\left[t_{0}, \infty\right) \rightarrow R$ with the sup norm. Define a subset $\Omega$ of $B C$ as follows:

$$
\Omega=\left\{y \in B C: 0 \leq y(t) \leq 1, t_{0} \leq t<\infty\right\} .
$$

Clearly $\Omega$ is a bounded, closed and convex subset of $B C$. Now we define a mapping $S$ on $\Omega$ as follows:

$$
(S y)(t)= \begin{cases}p \frac{v(t-\tau) y(t-\tau)}{v(t)}+\frac{1}{v(t)} \int_{T}^{t} q(s) \max _{u \in[s-\sigma, s]} v(u) y(u) d s+\frac{1}{2 v(t)}, & t \geq T \\ \frac{t}{T}(S y)(T)+\left(1-\frac{t}{T}\right), & t_{0} \leq t<T\end{cases}
$$


where $T$ is sufficiently large so that $t-\tau \geq t_{0}, t-\sigma \geq t_{0}, v(t) \geq 1$, and

$$
p \frac{v(t-\tau)}{v(t)}+\frac{1}{v(t)} \int_{T}^{t} q(s) \max _{u \in[s-\sigma, s]} v(u) d s \leq \frac{1}{2} \text { for } t \geq T .
$$

In fact, from (2), (3) and (4) it is easy to see that

$$
\frac{v(t-\tau)}{v(t)} \rightarrow 0 \text { and } \frac{\int_{t_{0}}^{t} q(s) \max _{u \in[s-\sigma, s]} v(u) d s}{v(t)} \rightarrow 0 \text { as } t \rightarrow \infty
$$

which shows that (6) is true for large $t$. Thus we have $S \Omega \subset \Omega$. Let $y_{1}$ and $y_{2}$ be two functions in $\Omega$. Then

$$
\begin{aligned}
\left|\left(S y_{2}\right)(t)-\left(S y_{1}\right)(t)\right| \leq & p \frac{v(t-\tau)}{v(t)}\left|y_{2}(t-\tau)-y_{1}(t-\tau)\right| \\
& +\frac{1}{v(t)} \int_{T}^{t} q(s) \max _{u \in[s-\sigma, s]} v(u)\left|y_{2}(u)-y_{1}(u)\right| d s \\
\leq & \frac{1}{2}\left\|y_{2}-y_{1}\right\|, t \geq T,
\end{aligned}
$$

and

$$
\begin{aligned}
\left\|S y_{2}-S y_{1}\right\| & =\sup _{t \geq t_{0}}\left|\left(S y_{2}\right)(t)-\left(S y_{1}\right)(t)\right| \\
& =\sup _{t \geq T}\left|\left(S y_{2}\right)(t)-\left(S y_{1}\right)(t)\right| \leq \frac{1}{2}\left\|y_{2}-y_{1}\right\|, \quad t \geq T
\end{aligned}
$$

which shows that $S$ is a contraction on $\Omega$. Hence there is a function $y \in \Omega$ such that $S y=y$. That is

$y(t)= \begin{cases}p \frac{v(t-\tau) y(t-\tau)}{v(t)}+\frac{1}{v(t)} \int_{T}^{t} q(s) \max _{u \in[s-\sigma, s]} v(u) y(u) d s+\frac{1}{2 v(t)}, & t \geq T \\ \frac{t}{T} y(T)+\left(1-\frac{t}{T}\right), & t_{0} \leq t<T .\end{cases}$

Obviously $y(t)>0$ for $t \geq t_{0}$. Set $x(t)=v(t) y(t)$. Then

$$
x(t)-p x(t-\tau)=\int_{T}^{t} q(s) \max _{u \in[s-\sigma, s]} x(u) d s+\frac{1}{2}, t \geq T .
$$

Therefore, $x(t)$ is a positive solution of (1) for $t \geq T$. The proof is complete.

In order to go a step further, we first give several preparatory results. The first lemma can be found in [5] or [6].

Lemma 2.2. Let $x, z \in C\left(\left[t_{0}, \infty\right), R\right)$ satisfy

$$
z(t)=x(t)-p x(t-\tau), t \geq t_{0}+\max \{0, \tau\},
$$

where $p, \tau \in R$. Assume that $x$ is bounded on $\left[t_{0}, \infty\right)$ and $\lim _{t \rightarrow \infty} z(t)=l$ exists. Then the following statements hold: 
(i) If $p=1$, then $l=0$;

(ii) If $p \neq \pm 1$, then $\lim _{t \rightarrow \infty} x(t)$ exists.

Lemma 2.3. Assume that $p \geq 0$ and let $x(t)$ be a positive solution of equation (1) and $x(t)-p x(t-\tau) \geq 0$. Then $x(t)$ satisfies either $(a)$ or $(b)$ :

$$
\text { (a) } \lim _{t \rightarrow \infty} x(t)=L \neq 0 \text {, (b) } \lim _{t \rightarrow \infty} x(t)=\infty \text {. }
$$

ProOF. Let $x(t)$ be a positive solution of equation (1). Set $z(t)=x(t)-$ $p x(t-\tau)$. Then $z(t) \geq 0$ and $z^{\prime}(t) \geq 0$ and is not identically zero. Hence $0<\lim _{t \rightarrow \infty} z(t)=l \leq \infty$. If $l=\infty$, then $x(t) \geq z(t) \rightarrow \infty$, as $t \rightarrow \infty$, i.e., (b) holds.

If $l<\infty$ and $x(t)$ is bounded, Lemma 2.2 implies that $\lim _{t \rightarrow \infty} x(t)$ exists when $p \neq 1$. But $\lim _{t \rightarrow \infty} x(t)=0$ is impossible. When $p=1$, Lemma 2.2 implies $l=0$. This is a contradiction.

If $l<\infty$ and $x(t)$ is unbounded, then there exists $\left\{t_{n}\right\}$ such that $x\left(t_{n}\right)=$ $\max _{t \leq t_{n}} x(t) \rightarrow \infty$ as $n \rightarrow \infty$. For $p \in(0,1), z\left(t_{n}\right) \geq x\left(t_{n}\right)(1-p) \rightarrow \infty$ as $n \rightarrow \infty$, which contradicts the boundedness of $z$. For $p=1, x(t) \geq$ $x(t-\tau)+l / 2 \geq \ldots \geq x(t-n \tau)+n l / 2 \rightarrow \infty$ as $n \rightarrow \infty$. For $p>1$, we have

$$
x(t) \geq p x(t-\tau) \geq \ldots \geq p^{n} x(t-n \tau)
$$

which implies that (b) holds. The proof is complete.

REMARK 2.4. In Lemma 2.3, the condition $x(t)-p x(t-\tau) \geq 0$ is necessary. For example, we consider the equation

$$
(x(t)-2 x(t-1))^{\prime}=(2 e-1) e^{-t}\left\{\max _{s \in[t-1, t]}\left(\varphi(s)+e^{-s}\right)\right\}^{-1} \max _{s \in[t-1, t]} x(s),
$$

where

$$
\varphi(t)=\left\{\begin{array}{ll}
2^{n}(t-n), & t \in\left[n, n+\frac{1}{2}\right] \\
2^{n}(n+1-t), & t \in\left[n+\frac{1}{2}, n+1\right]
\end{array} \quad n=0,1, \ldots\right.
$$

It has an eventually positive solution $x(t)=\varphi(t)+e^{-t}$. In fact, $x(t)$ satisfies $\limsup \operatorname{sum}_{t \rightarrow \infty} x(t)=\infty$ and $\liminf _{t \rightarrow \infty} x(t)=0$. As if $\sigma=0$, the condition $x(t)-p x(t-\tau) \geq 0$ is also necessary. Consider the equation

$$
(x(t)-2 x(t-1))^{\prime}=(2 e-1) e^{-t}\left(\varphi(t)+e^{-t}\right)^{-1} x(t) .
$$

It has also positive solution $x(t)=\varphi(t)+e^{-t}$. Thus, we see that Lemma 3.5.1 in [5] is false. result.

By Theorem 2.1 and Lemma 2.3, we obtain immediately the following

THEOREM 2.5. Based on the range of $p$ we have the conclusion:

(i) If $p=1$, equation (1) has an unbounded positive solution $x(t)$ satisfying (b); 
(ii) If $p>1$, equation (1) has an unbounded positive solution $x(t)$ which tends to infinity exponentially;

(iii) If $0 \leq p<1$, and

$$
\int_{t_{0}}^{\infty} q(s) d s=\infty
$$

equation (1) has an unbounded positive solution.

ProOF. By (7), we have clearly

$$
x(t)-p x(t-\tau)>0 \text { for } t \geq T .
$$

(i) and (iii) are clear. In the case that $p>1$ we have

$$
x(t) \geq p x(t-\tau) \geq \ldots \geq p^{n} x(t-n \tau),
$$

or

$$
x(t) \geq x\left(t_{0}\right) \exp \left(\mu\left(t-t_{0}\right)\right), \text { for } t \geq t_{0},
$$

where $\mu=\frac{\ln p}{\tau}>0$, which shows that (ii) is true. The proof is complete.

Theorem 2.6. Assume that $p \neq 1$ and $\int_{t_{0}}^{\infty} q(s) d s<\infty$. Then equation (1) has a bounded positive solution.

The proof of Theorem 2.6 is similar to the proof of Theorem 3.2.11 in [5]. It will be omitted.

REMARK 2.7. For eventually negative solutions of equation (1), we can also obtain similar results. They are omitted.

\section{Asymptotic Behavior of Nonoscillatory Solutions}

In this section, we will obtain asymptotic properties of nonoscillatory solutions of equation (1).

Theorem 3.1. Suppose that (8) holds and $0 \leq p<1$. If $x(t)$ is a nonoscillatory solution of equation (1), then either $\lim _{t \rightarrow \infty}|x(t)|=\infty$ or $\lim _{t \rightarrow \infty} x(t)=0$.

Proof. Let $x(t)$ be an eventually positive solution of (1) and set

$$
z(t)=x(t)-p x(t-\tau) .
$$

Then we have either $z(t)>0$ or $z(t)<0$. If $z(t)>0$ holds, then there exists $c>0$ such that $x(t) \geq z(t)>c$. Integrating (1) we have

$$
z(t)=\int_{T}^{t} q(s) \max _{u \in[s-\sigma, s]} x(u) d s+z(T) \geq c \int_{T}^{t} q(s) .
$$

It is clear that $\lim _{t \rightarrow \infty} z(t)=\infty$. Thus, we have $\lim _{t \rightarrow \infty} x(t)=\infty$.

If $z(t)<0$ holds, then $p \neq 0$ and $\lim _{t \rightarrow \infty} z(t)=L \leq 0$ is finite. Integrat- 
ing (1) we have

$$
L-z(t)=\int_{t}^{\infty} q(s) \max _{u \in[s-\sigma, s]} x(u) d s .
$$

Thus, we have $\liminf \operatorname{in}_{t \rightarrow \infty} x(t)=0$. Note that $-p x(t-\tau)<z(t)$, we have $\lim _{t \rightarrow \infty} z(t)=0$. On the other hand, $x(t)<p x(t-\tau)<x(t-\tau)$ implies that $x(t)$ is bounded above. By Lemma 2.2, we get that $\lim _{t \rightarrow \infty} x(t)$ exists. Let $\lim _{t \rightarrow \infty} x(t)=l$. In view of $(9)$, we get that

$$
0=\lim _{t \rightarrow \infty} x(t)-p \lim _{t \rightarrow \infty} x(t-\tau)=l(1-p)
$$

which implies $l=0$. The case where $x(t)$ is eventually negative is proved in a similar way. The proof is complete.

Corollary 3.2. Suppose that (8) holds and $0 \leq p<1$. If $x(t)$ is a bounded nonoscillatory solution of equation (1), then $\lim _{t \rightarrow \infty} x(t)=0$.

Theorem 3.3. Suppose that (8) holds and $p>1$. If $x(t)$ is a bounded nonoscillatory solution of equation (1), then $\lim _{t \rightarrow \infty} x(t)=0$.

Proof. Let $x(t)$ be a bounded eventually positive solution of (1) and $z(t)$ is defined by (9). Then $z(t)>0$ is impossible. Indeed, then we have $\lim _{t \rightarrow \infty} x(t)=\infty$ which is a contradiction. Thus, we have $z(t)<0$. In view of the proof of Theorem 3.1, we have $\lim _{t \rightarrow \infty} z(t)=0$. Similarly, we have

$$
0=\lim _{t \rightarrow \infty} x(t)-p \lim _{t \rightarrow \infty} x(t-\tau)=l(1-p)
$$

which implies $l=0$. The case where $x(t)$ is eventually negative is similarly proved. The proof is complete.

Theorem 3.4. Suppose that $p \geq 1$ and $0<q \leq q(t)$. If $x(t)$ is an eventually positive solution of equation (1) then either $\lim _{t \rightarrow \infty} x(t)=\infty$ or $\lim _{t \rightarrow \infty} x(t)=0$.

Proof. If $z(t)>0$, we have similarly $\lim _{t \rightarrow \infty} x(t)=\infty$. If $z(t)<0$ holds, then $\lim _{t \rightarrow \infty} z(t)=L \leq 0$ is finite. Suppose that $x(t)$ does not tend to zero as $t \rightarrow \infty$. Then $c=\lim \sup _{t \rightarrow \infty} x(t)>0$ (if $x(t)$ is unbounded, set $c$ to be an arbitrary positive constant). There exists a sequence $\left\{t_{n}\right\}_{1}^{\infty}$ such that $t_{n+1}-t_{n}>\sigma$ and $x\left(t_{n}\right)>c / 2$ for each $n \in N$. Thus the inequality

$$
\max _{s \in[t-\sigma, t]} x(s)>\frac{c}{2}, t \in\left[t_{n}, t_{n}+\sigma\right], n \geq N,
$$

holds, and

$$
\int_{t_{n}}^{t_{n}+\sigma} q(s) \max _{u \in[s-\sigma, s]} x(u) \geq \frac{c q \sigma}{2} .
$$


From the definition of the sequence $\left\{t_{n}\right\}$ and integrating (1), we have

$$
\begin{aligned}
L-z\left(t_{0}\right) & =\int_{t_{0}}^{\infty} q(s) \max _{u \in[s-\tau, s]} x(u) d s \\
& \geq \sum_{n=1}^{\infty} \int_{t_{n}}^{t_{n}+\sigma} q(s) \max _{u \in[s-\sigma, s]} x(u) \\
& \geq \sum_{n=1}^{\infty} \frac{c q \sigma}{2}=\infty .
\end{aligned}
$$

This is a contradiction. The proof is complete.

REMARK 3.5. Theorem 3.4 is not valid if $x(t)$ is an eventually negative solution of equation (1) as the following example shows.

Example 3.6. Consider the equation

$$
(x(t)-2 x(t-1))^{\prime}=(2 e-1) e^{-t}\left\{\min _{s \in[t-1, t]}\left(\varphi(s)+e^{-s}\right)\right\}^{-1} \max _{s \in[t-1, t]} x(s),
$$

where

$$
\varphi(t)=\left\{\begin{array}{ll}
2^{n}(t-n), & t \in\left[n, n+\frac{1}{2}\right] \\
2^{n}(n+1-t), & t \in\left[n+\frac{1}{2}, n+1\right]
\end{array} \quad n=0,1, \ldots .\right.
$$

It is easy to check that

$$
(2 e-1) e^{-t}\left\{\min _{s \in[t-1, t]}\left(\varphi(s)+e^{-s}\right)\right\}^{-1} \geq 2 e-1>0,
$$

so, the assumptions of Theorem 3.4 are satisfied. A straightforward verification yields that the function $x(t)=-\varphi(t)-e^{-t}$ is an eventually negative solution of (10). Moreover, since $x(n)=-e^{-n}$ and $x\left(n+\frac{1}{2}\right)=-2^{n-1}-e^{-n-\frac{1}{2}}$, $\lim \sup _{t \rightarrow \infty} x(t)=0$ and $\liminf _{t \rightarrow \infty} x(t)=-\infty$.

Corollary 3.7. Suppose that $p=1$ and $0<q \leq q(t)$. Then a bounded eventually positive solution $x(t)$ of equation (1) satisfies $\lim _{t \rightarrow \infty} x(t)=0$.

REMARK 3.8. Corollary 3.7 is not valid if $x(t)$ is a bounded eventually negative solution of equation (1).

EXAmple 3.9. Consider the equation

(11) $(x(t)-x(t-1))^{\prime}=(e-1) e^{-t}\left\{\min _{s \in[t-1, t]}\left(\psi(s)+e^{-s}\right)\right\}^{-1} \max _{s \in[t-1, t]} x(s)$,

where $\psi(t)$ is a 1-periodic function defined by the equality

$$
\psi(t)=\left\{\begin{array}{ll}
t, & t \in\left[0, \frac{1}{2}\right] \\
1-t, & t \in\left[\frac{1}{2}, 1\right]
\end{array} .\right.
$$


It has an eventually negative solution $x(t)=-\psi(t)-e^{-t}$ and

$$
\lim \sup _{t \rightarrow \infty} x(t)=0 \text { and } \lim \inf _{t \rightarrow \infty} x(t)=-1 / 2 .
$$

EXAMPLE 3.10. Consider the equation

$$
(x(t)-p x(t-\tau))^{\prime}=q \max _{s \in[t-\sigma, t]} x(s),
$$

where $q=\left(p e^{\tau}-1\right) / e^{\sigma}$. When $e^{-\tau}<p<1$, (12) satisfies all conditions of Theorem 3.1 or Corollary 3.2. If $p>1$, it satisfies all conditions of Theorem 3.3; and if $p=1$, all conditions of Corollary 3.7 hold. In fact, (12) has a positive solution $x(t)=e^{-t}$.

\section{Oscillation Results}

In view of Section 2, we have known that equation (1) has an eventually positive solution when $p \geq 0$. Thus, we will discuss first its oscillation for $p<0$.

Theorem 4.1. Assume that $p<0, p \neq-1$ and the condition (8) holds. Then every bounded solution of equation (1) is oscillatory.

Proof. Let $x(t)>0$ be a bounded positive solution of (1). Set $z(t)=$ $x(t)-p x(t-\tau)$, then $z^{\prime}(t) \geq 0$. Thus $\lim _{t \rightarrow \infty} z(t)=l$ exists and $l>0$. For $p \neq-1$, by Lemma 2.2 , there exists $\lim _{t \rightarrow \infty} x(t)$. From (1) we have

$$
\int_{t_{0}}^{\infty} q(t) \max _{s \in[t-\sigma, t]} x(s) d t<\infty .
$$

In view of (8), this implies that $\lim _{t \rightarrow \infty} x(t)=0$ and so by $(9) l=(1-p) 0=0$. This contradicts $l>0$. The proof in the case of eventually negative solution is similar and will be omitted.

In fact, when $p<0$ and $q=1-p e^{-\tau}$, a straightforward verification yields that the function $x(t)=e^{t}$ is an eventually positive solution of equation (12). Furthermore, we know that (1) has also an unbounded eventually positive solution when $p=-1$ as if the condition (8) holds. But we have also the following result.

Theorem 4.2. Let $p=-1$. Assume $\int_{E} q(t) d s=\infty$ for every closed subset $E$ of $\left[t_{0}, \infty\right)$ whose intersection with every interval of the form $[t, t+2 \tau]$, $t_{0} \leq t<\infty$, has a measure not less than $\tau$. Then every bounded solution of equation (1) is oscillatory.

Its proof is similar to Theorem 3.5.4 in [5]. Thus it is omitted. In case $0 \leq p$, we have the following results. 
Theorem 4.3. Assume that $p \geq 0, p \neq 1$ and the condition (8) holds. Then every bounded solution of equation (1) is either oscillatory or tends to zero as $t \rightarrow \infty$.

In view of Theorem 3.1 or Theorem 3.3, we know immediately that Theorem 4.3 is valid.

Theorem 4.4. Assume that $p>0, p \neq 1$ and the condition (8) holds. Suppose further that

$$
z^{\prime}(t)+\frac{1}{p} q(t) z(t+\tau-\sigma) \geq 0
$$

has no eventually positive solution and

$$
z^{\prime}(t)+\frac{1}{p} q(t) z(t+\tau) \leq 0
$$

has no eventually negative solution. Then every bounded solution of equation (1) is oscillatory.

Indeed, from the proof of Theorem 4.3, we see that if $x(t)$ is a bounded positive solution of (1), then $z(t)<0$ eventually. Note that $x(t)>-z(t+\tau) / p$. Substituting it into (1) we obtain

$$
z^{\prime}(t)+\frac{1}{p} q(t) z(t+\tau-\sigma) \geq 0 .
$$

This is a contradiction. For a bounded negative solution, we can obtain similarly contradiction.

Theorem 4.5. Assume that $p=1$ and the condition (8) holds. Suppose further

$$
y^{\prime \prime}(t) \geq \frac{1}{\tau} q(t+\tau) y(t+\tau-\sigma)
$$

has no any eventually positive solution and

$$
y^{\prime \prime}(t-\tau) \leq \frac{1}{\tau} q(t) y(t)
$$

has no any eventually negative solution. Then every bounded solution of equation (1) oscillates.

Proof. Let $x(t)$ be a bounded eventually positive solution of (1) and set $z(t)=x(t-\tau)-x(t)$. Then $z(t)>0$ and $z^{\prime}(t) \leq 0$ eventually. Thus, we have 
also

$$
\begin{aligned}
x(t) & =x(t+\tau)+z(t+\tau) \\
& \geq x(t+\tau)+\frac{1}{\tau} \int_{t+\tau}^{t+2 \tau} z(s) d s \\
& \geq \frac{1}{\tau} \int_{t+\tau}^{\infty} z(s) d s .
\end{aligned}
$$

Set

$$
y(t)=\frac{1}{\tau} \int_{t+\tau}^{\infty} z(s) d s
$$

Substituting it into (1) we obtain

$$
y^{\prime \prime}(t) \geq \frac{1}{\tau} q(t+\tau) y(t+\tau-\sigma)
$$

which is a contradiction.

If $x(t)$ is a bounded eventually negative solution of (1), we have $z(t)<0$ and $z^{\prime}(t) \geq 0$ eventually. Thus, we have also

$$
x(t)=x(t+\tau)+z(t+\tau) \leq \frac{1}{\tau} \int_{t+\tau}^{\infty} z(s) d s .
$$

$y(t)$ is defined by (13), and substituting it into (1) we have

$$
y^{\prime \prime}(t-\tau) \leq \frac{1}{\tau} q(t) y(t) .
$$

The proof is complete.

\section{REFERENCES}

[1] E.P. Popov, Automatic Regulation and Control, Nauka, Moscow, 1966.

[2] D. Bainov, V. Petrov and V. Proicheva, Oscillation of neutral differential equations with "maxima", Revista Math. 8(1) (1995), 171-180.

[3] B.G. Zhang and G. Zhang, Qualitative properties of functional differential equations with "maxima", Rocky Mountain J. Math. 29(1) (1999), 357-367.

[4] G. Zhang and S.S. Cheng, Asymptotic stability of nonoscillatory solutions of nonlinear neutral differential equations involving the maximum function, International J. Applied Math. 7 (1999), 771-779.

[5] L.H. Erbe. Q. Kong and B.G. Zhang, Oscillation Theory for Functional Differential Equations, Marcel Dekker, Inc., New York, 1995.

[6] I. Gyori and G. Ladas, Oscillation Theory of Delay Differential Equations with Applications, Clarendon Press, Oxford, 1991. 
G. Zhang

Department of Mathematics

Qingdao Technological University

11, Fushun Road, Qingdao 266033

P. R. China

E-mail: dtguangzhang@yahoo.com.cn

M. Migda

Institute of Mathematics

Faculty of Electrical Engineering

Poznań University of Technology

Piotrowo 3a, 60-965 Poznań

Poland

E-mail: mmigda@math.put.poznan.pl

Received: 3.6.2004. 